\title{
A study of the earthquake-resistance of masonry buildings constructed with local materials
}

\author{
A. Koçak ${ }^{1}$, T. Karaca ${ }^{1}$, B. Pazır ${ }^{1}$, B. Zengin ${ }^{2} \&$ A. Kalyoncuoğlu ${ }^{3}$ \\ ${ }^{I}$ Department of Civil Engineering, Yildiz Technical University, Turkey \\ ${ }^{2}$ Vocational School, Istanbul Gelisim University, Istanbul, Turkey \\ ${ }^{3}$ Department of Civil Engineering, Istanbul Gelisim University, Turkey
}

\begin{abstract}
Masonry buildings constitute most of the buildings in our country. Although they are used both in rural areas and city centres, supplying the material around the building area is an important factor. Thus, especially in the countryside these types of buildings are more preferable but there may be problems under dynamic loads such as horizontal loads and earthquake when some rules in architecture are not obeyed.

During this study, many different masonry buildings in many different regions of Turkey were discussed, the materials using in those buildings were examined, and their earthquake resistances were evaluated by using the finite elements technique in terms of seismicity and building models. As a result of the study, the earthquake resistance of buildings which were constructed with different materials in different regions of Turkey were determined, and the reinforcements of buildings having a lack of earthquake resistance and their situations after reinforcement were determined.

Keywords: masonry building, earthquake analysis, repair and strengthening.
\end{abstract}

\section{Introduction}

Turkey is located over an earthquake region, and the buildings, especially in rural areas, are constructed with materials which vary depending on the conditions of the region. Most of those structures are not earthquake-resistant. When evaluating the damage to masonry buildings in recent earthquakes in Turkey, those kinds of buildings that are not earthquake-resistant can be seen 
quite obviously. The movements of masonry buildings during an earthquake and the analysis of those buildings are complicated situations. Almost in every country, studies are conducted in order to determine the behaviour of masonry buildings being used as a historical place or a house, and to determine the specifications of their materials [1-8]. By determining the post-earthquake damage of those structures, some analytical and experimental studies can be conducted in order to determine the bearing of walls and the specifications of materials for preventing the newly-constructed structures from the effects of an earthquake [9-12]. Also when strengthening the weak structures in order to make them earthquake-resistant, many researchers have conducted studies about the reinforcement and strengthening of historical buildings in particular [13-16, 18]. The earthquake-resistant design of both ferro-concrete and masonry structures can be defined as constructing buildings which are resistant to earthquakes and forces on structures. In order to design the structures as earthquake resistant, it is important to know the forces which arise during an earthquake and to examine efficiently the behaviour of structures under those effects [19-23].

During this study, architectural plans, material characteristics and carrier systems of masonry structures constructed in different regions of Turkey were examined. The behaviours of structures under earthquake loads were determined by using the finite elements method. By reinforcing the structures with earthquake-resistance deficiency, their conditions before and after reinforcements were compared.

\section{The types of masonry structures in Turkey and the materials used}

The materials being used around Turkey are wooden in Blacksea and Marmara, adobe in Central Anatolia, and stone in Eastern Turkey. Without changes between regions in terms of plan, the houses where internal privacy rules are not breached can be seen [24]. In the introduction part of Aksoy's article "The establishment principles of middle space" [25], he mentioned that although Turkey consists of 7 geographical regions, it can be reduced to 3 climate regions practically because of similar and same data. In the chapter on "Climate Regions of Anatolia, and Effects of Those Regions on Culture and Art Activities", he classified those 3 climate regions as 1 . The Mediterranean Region: South Anatolia and Aegean regions, 2. Temperate Region: Marmara and North Anatolia, 3. Continental Climate Region: Plateaus of Anatolia. According to Aksoy [25], the information of the 3 main climate regions creates 3 different patterns.

In that order, the structures are classified as of stone, wooden, and adobe. Their distribution into regions is drawn as follows; stone-made structures are seen in the southeast, wooden-made ones are seen in Marmara and Aegean regions, adobe-made ones are seen in high central plateaus, and solid wood-made ones are seen in North Anatolia and central plateaus' parts with large forests [26]. It was seen that some of those materials may be used together in some 
regions (Fig. 1). The materials of masonry structures include mostly inorganic materials having no metal properties, from clay-based adobe to natural stone and bricks. But the most frequently used materials are stone, bricks and adobe. Those structures are stone, logging, adobe, briquette, timber-work, and hybrid masonry structures [27].

a.
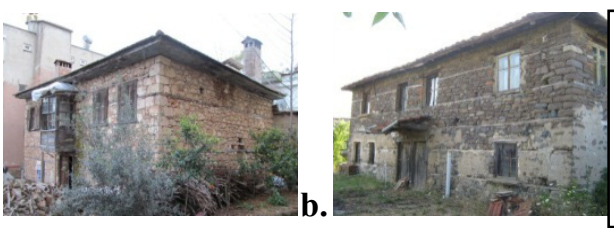

Materials used in stone building: Appropriate-shaped stones around an area, bonding timbers and laying, and mud-halm mixture (as a bonding element).

c.
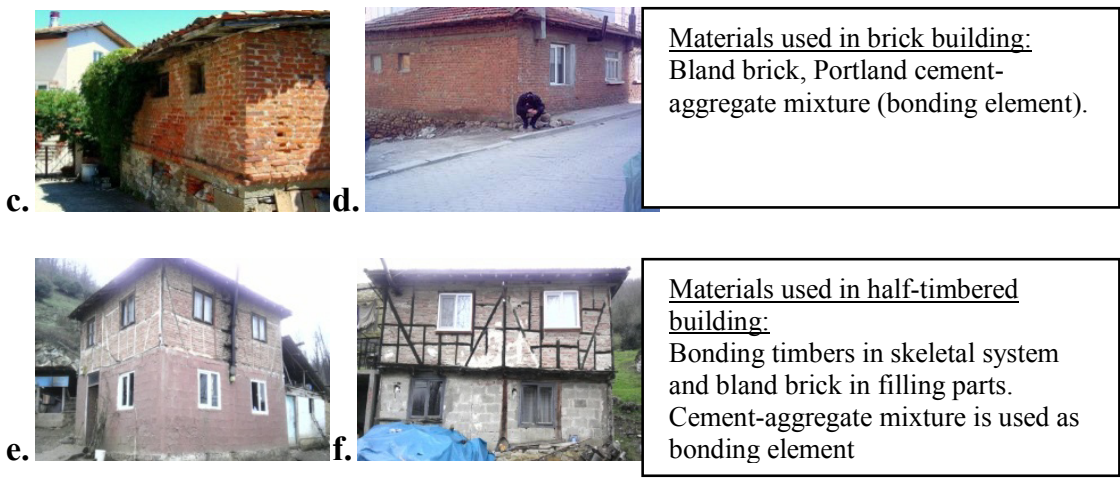

Materials used in half-timbered building:

Bonding timbers in skeletal system and bland brick in filling parts.

Cement-aggregate mixture is used as bonding element
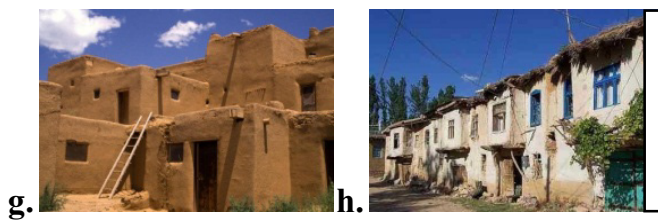

Materials used in adobe building: Adobe which is obtained by well mixing the mud and halm. Wood is used in windows and doors.

Figure 1: Masonry structure samples from different regions.

Additional to uniform ones, mixed structures are also used in our constructions according to conditions of regions. We see that wood, adobe, and stone materials are used in traditional houses of Burdur at most. Because of its easy workmanship and its efficient resistance to heat and cold, adobe is widely used in the region [28]. Duplex or triplex masonry structures, which have plane frontals and sometimes courts, do not offend the eye within the concept of the Diyarbakır Suriçi region. The most important problem of Suriçi is the existence of column-free and groundworkless houses with different wall thicknesses and more than 3 floors [29]. The main construction element of houses in the Yozgat region is stone. Stone is used in groundworks and the walls of the house as a carrier. Also depending on usage field, it is used as a decoration element in garden walls and chimneys. Soil is the element bonding the walls. It is used in walls and layings. Wood is the second most frequently used material in the construction of houses [30]. Also, when analysing the traditional architecture of 
village houses in Turkey, it is seen that the plan is very simple and there are 2 or 3 floors in the highest structures, and that wall thicknesses are between 20 and $55 \mathrm{~cm}$. There are some plans and photos of village houses in Figure 2.

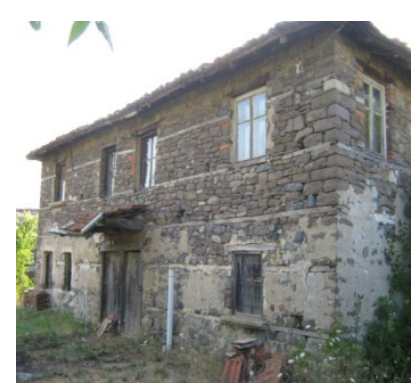

a)

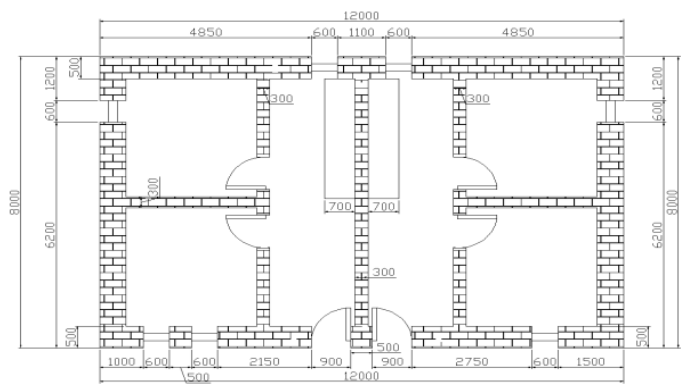

b)

Figure 2: a) Photo and ground floor plan of stone masonry house in the Edirne region. b) Ground floor plan of masonry structure.

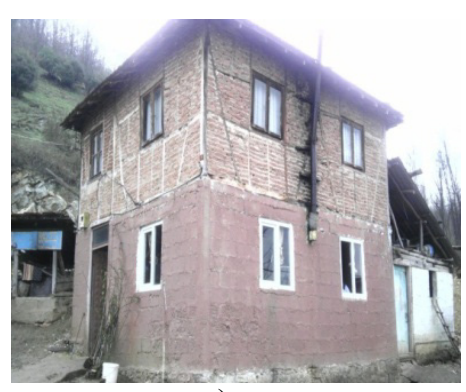

a)

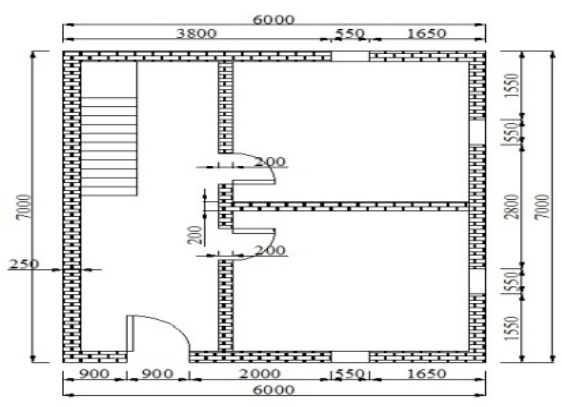

b)

Figure 3: a) A structure with briquette masonry walls in ground floor and half-timbered walls in upper floor in the Sakarya region. b) Plan of ground floor of structure.

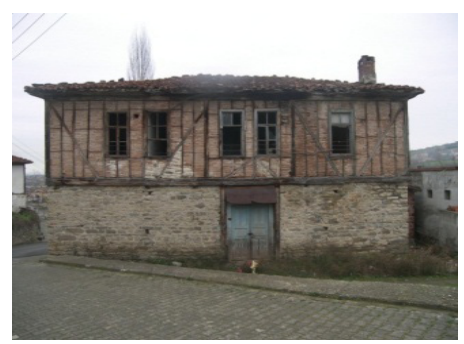

a)

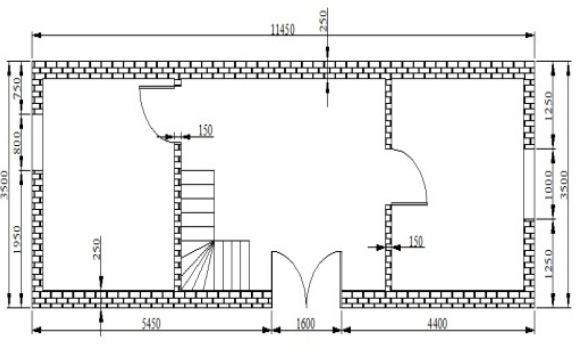

b)

Figure 4: a) A structure with stone masonry walls in ground floor and halftimbered walls in upper floor in the Samsun region. b) Plan of ground floor of structure. 


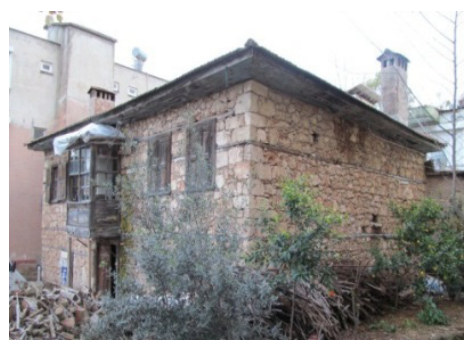

a)

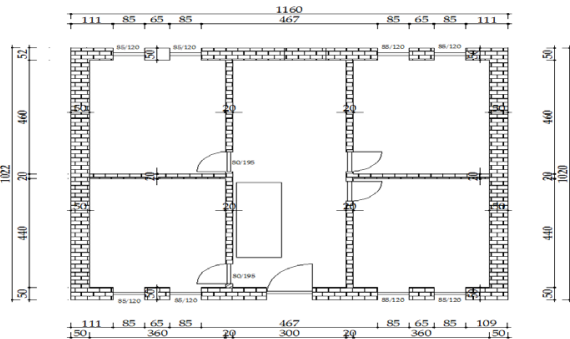

b)

Figure 5: a) Stone masonry structure in the Antalya region. b) Plan of ground floor of structure.

\section{Calculation of masonry buildings according to Turkish seismic code}

The calculations of masonry buildings' resistance to earthquake were performed according to the $5^{\text {th }}$ chapter of the Turkish Seismic Code-TSC (2007) by determining the shear stress of earthquake on horizontal joints of walls. Before calculation of shear stress, the vertical stresses on walls were calculated, and they were evaluated as to whether they conform within the desired limits or not. Wall safety stress was calculated according to block brick with vertical pores, and the wall safety stresses were determined according to values given by TSC (2007) $[31,32]$. Calculated wall pressure safety stresses were decreased according to their fineless degrees. In order to calculate the shear stresses on walls, shear force on walls was calculated through the ETABS 9.7.2 version by considering the floor torsional moment. By dividing the earthquake force on the wall with a horizontal cross-sectional area of the wall, the shear stress was calculated. That value was compared with wall shear stress $\tau_{e m}$ which was found via Equation (1). $\tau_{e m}$ indicates the wall shear security stress, $\tau_{o}$ indicates the wall cracking shear stress, $\mu$ indicates the friction coefficient, and $\sigma$ indicates the precalculated horizontal wall tension. Three different models were used in the calculations. A first-degree seismic zone was handled in the analyses, and effective ground acceleration was accepted as $0.4 \mathrm{~g}$. The building importance coefficient was taken as 1, class of soil class was chosen as Z1 according to TSC.

$$
\tau_{e m}=\tau_{o}+\mu \sigma
$$

The material and geometrical specifications of the wall, bond beam and used in model are given in Table 1. The thickness of the wall was calculated as $10 \mathrm{~cm}$ in all of the models. Likewise, the intersection sizes of bond beams were arranged as $50 \times 50 \mathrm{~cm}$ and $50 \times 30 \mathrm{~cm}$, according to the thicknesses of the walls. 
Table 1: The mechanic specifications of the wall, bond beam and laying used in the model.

\begin{tabular}{|c|c|c|c|c|c|c|c|}
\hline Type & Material & $\begin{array}{c}\text { Mass } \\
(\mathrm{kg})\end{array}$ & $\begin{array}{c}\text { Weight } \\
(\mathrm{kg} \\
\left.\mathrm{ms}^{2}\right)\end{array}$ & $\begin{array}{c}\text { Model } \\
\text { Pressure } \\
\text { Resistance } \\
(\mathrm{MPa})\end{array}$ & $\begin{array}{c}\text { Elasticity } \\
\text { module } \\
(\mathrm{MPa})\end{array}$ & $\begin{array}{c}\text { Poisson } \\
\text { Rate }\end{array}$ & $\begin{array}{c}\text { Shear } \\
\text { Module } \\
(\mathrm{MPa})\end{array}$ \\
\hline Wall & Cliff stone & 2.54 & 25 & 10 & 2000 & 0.15 & 870 \\
\hline Laying & $\begin{array}{c}\text { Ferro- } \\
\text { concrete }\end{array}$ & 2.54 & 25 & 16.7 & 28000 & 0.2 & 11666 \\
\hline $\begin{array}{c}\text { Bond } \\
\text { Beam }\end{array}$ & $\begin{array}{c}\text { Ferro- } \\
\text { concrete }\end{array}$ & 2.54 & 25 & 16.7 & 28000 & 0.2 & 11666 \\
\hline
\end{tabular}

Also the unsafe buildings which did not satisfy the TSC2007, were retrofitted with steel profiles and FRP. Considering the wall thicknesses, the behaviour of the weak walls were analysed by placing horizontal and vertical 50x50 and 30x50 bond beams with at least $4 \mathrm{~m}$ intervals (according to Turkish Seismic Code). In another model, besides the 50x50 and 30x50 horizontal and vertical bond beams, the weak walls were reinforced with UNP 400 cross-sectional steel and FRP implementation. In another model, reinforcement was performed with repair mortar. That implementation was investigated in 20 and $50 \mathrm{~cm}$-thickness walls according to Turkish Seismic Code. And finally, reinforcements were

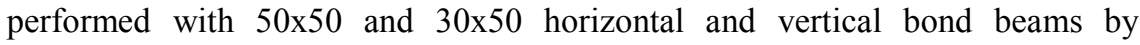
assuming the thickness of all walls as $20 \mathrm{~cm}$, then the earthquake bearing of buildings were analysed.

\section{Analysis results}

At the end of the analyses of models, all the data were obtained, and shear force on walls and shear stresses were calculated according to TSE. Obtained vertical stress values, shear stresses and calculated security stresses are given in Tables 2 and 3 (for 2-storey structures) and Table 4 (for 3-storey structures). All of the calculations were performed according to the Turkish Seismic code. "V" sign denotes that the structure is safe according to the code, and " $\mathrm{X}$ " sign denotes that the structure is not safe. The model images of the buildings are given in Figures 6-8. 
Table 2: Analysis results of the $1^{\text {st }}$ model ( $\tau$ : shear stress, $\tau$ em: safety coefficient y shear stress).

\begin{tabular}{|c|c|c|c|c|c|c|c|c|c|c|c|c|c|c|}
\hline \multirow{3}{*}{$\begin{array}{c}\text { Building Types } \\
\text { Wall Thickness } \\
\text { Wall No }\end{array}$} & \multicolumn{2}{|c|}{ Original } & \multicolumn{2}{|c|}{$50 \times 50$ Bond Beam } & \multicolumn{2}{|c|}{$30 \times 50$ Bond Beam } & \multicolumn{2}{|c|}{\begin{tabular}{|c|}
$50 x 50$ Bond \\
Beam + Cross \\
\end{tabular}} & \multirow{2}{*}{\multicolumn{2}{|c|}{\begin{tabular}{|c|}
$\begin{array}{c}\text { Repair Mortar +FRP } \\
\text { Reinforcement }\end{array}$ \\
$0.5 \mathrm{~m}$ \\
\end{tabular}}} & \multirow{2}{*}{\multicolumn{2}{|c|}{$\begin{array}{c}\begin{array}{c}\text { Repair Mortar +FRP } \\
\text { Reinforcement }\end{array} \\
\mathbf{0 . 2 ~} \mathbf{m}\end{array}$}} & \multirow{2}{*}{\multicolumn{2}{|c|}{$\begin{array}{c}\text { 30x50 Bond Beam } \\
0.2 \mathrm{~m}\end{array}$}} \\
\hline & \multicolumn{2}{|c|}{$0.5 \mathrm{~m}$} & \multicolumn{2}{|c|}{$0.5 \mathrm{~m}$} & \multicolumn{2}{|c|}{$0.5 \mathrm{~m}$} & \multicolumn{2}{|c|}{$0.5 \mathrm{~m}$} & & & & & & \\
\hline & $\tau$ & $\tau \mathrm{em}$ & $\tau$ & $\tau \mathrm{em}$ & $\tau$ & $\tau e \mathrm{~m}$ & $\tau$ & $\tau \mathrm{em}$ & $\tau$ & $\tau \mathrm{em}$ & $\tau$ & $\tau \mathrm{em}$ & $\tau$ & $\tau e \mathrm{~m}$ \\
\hline & & & & & & & & & & & 0,23 & & & \\
\hline & & & & & & & & &, 16 & & 0,18 & & & \\
\hline & & & & & & & & & & &, 20 & & & \\
\hline $\mathrm{P} 2 \mathrm{X} 1$ & & & & & & & & & & & $\mathrm{X}$ & & & \\
\hline & & & & & & & & & & & & & & \\
\hline & & & & & & & & & & & & & & \\
\hline & & & & & & & & & & & & & & \\
\hline & & & & & & & & & & & 0 & & & \\
\hline & & & & & & & & & & & $\sqrt{ }$ & & & \\
\hline PX1 & & $0,17 \sqrt{ }$ & & & & & & & & & & & & \\
\hline & & & & & & & & & & & $\sqrt{ }$ & & & \\
\hline & & & & & & & & & & & $\pi$ & & & \\
\hline & & & & & & & & & & & $\sqrt{ }$ & & & \\
\hline & & & & & & & & & & & & & & \\
\hline & & & & & & & & & & & $\sqrt{ }$ & & & \\
\hline & & $19 \sqrt{ }$ & & & & & & & & & $\sqrt{ }$ & & & \\
\hline & & & & & & & & & & & & & & \\
\hline & & & & & & & & & & & $\sqrt{ }$ & & & \\
\hline & & & & & & & & & 2 & 22 & 3 & & 02 & \\
\hline & & $20 \mathrm{~V}$ & & & & & & & 0,0 & 0,25 & 3 & $0,25 \mathrm{~V}$ & 01 & \\
\hline & & & & & & & & & & 0,24 & & $0,25 \sqrt{ }$ & & \\
\hline & & & & & & & & & & & $\sqrt{ }$ & & & \\
\hline & & & & & & & & & & & & & & \\
\hline & & $0,17 \sqrt{ }$ & 0,02 & & & $0,14 \sqrt{ }$ & & & 0,03 & 0,22 & 03 & $0,24 \mathrm{~V}$ & & \\
\hline & & $0,17 \sqrt{ }$ & & $0,13 \sqrt{ }$ & & $0,14 \sqrt{ }$ & & & 0,02 & 0,22 & 04 & $0,24 \mathrm{~V}$ & 02 &, $13 \mathrm{~V}$ \\
\hline & & & & & & $0,11 \sqrt{ }$ & & $0 \sqrt{1}$ & 0,10 & 0,15 & 0,13 & $0,16 \mathrm{~V}$ & 0,08 & $0,11 \sqrt{ }$ \\
\hline & & & & & & & & & & & & & 0,08 & \\
\hline P1XKY2-8 & 0,10 & $0,19 \sqrt{ }$ & 0,06 & $0,14 \sqrt{ }$ & 0,07 & $0,15 \sqrt{ }$ & 0,04 & $0,14 \sqrt{ }$ & 0,10 & 0,24 & 0,12 & $0,25 \mathrm{~V}$ & 0,07 & \\
\hline
\end{tabular}
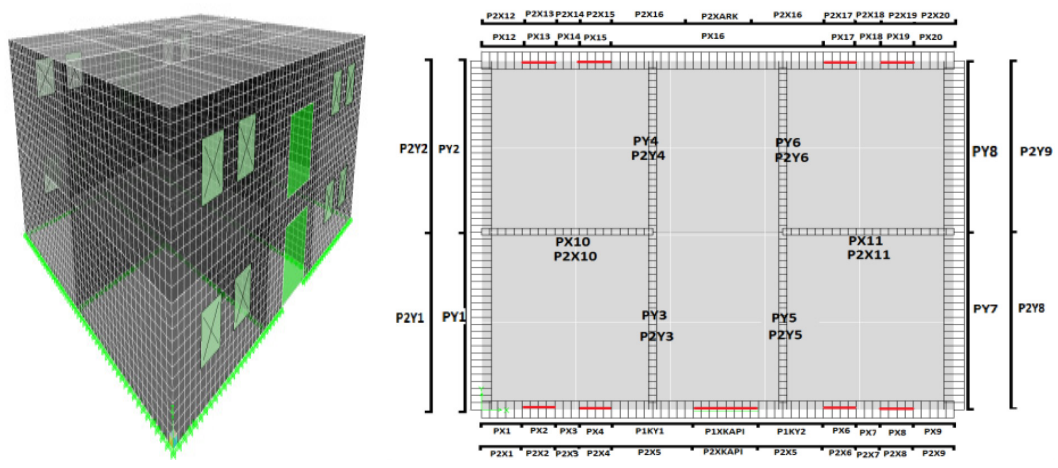

Figure 6: Image and plan of the $1^{\text {st }}$ model. 
Table 3: $\quad$ Analysis results of the $2^{\text {nd }}$ model.

\begin{tabular}{|c|c|c|c|c|c|c|c|c|c|c|c|c|c|c|c|c|c|c|c|c|}
\hline B uilding Types & \multicolumn{3}{|c|}{ Original } & \multicolumn{3}{|c|}{;0x50 Bond Bean } & \multicolumn{3}{|c|}{$30 \times 50$ Bond Bean } & \multicolumn{3}{|c|}{$\begin{array}{c}\text { 50x50 Bond } \\
\text { Beam + Cross } \\
\text { UNP }\end{array}$} & \multicolumn{2}{|c|}{$\begin{array}{c}\text { Repair } \\
\text { Mortar +FRP }\end{array}$} & \multicolumn{3}{|c|}{$\begin{array}{c}\text { Repair } \\
\text { Mortar +FRP }\end{array}$} & \multicolumn{3}{|c|}{$\begin{array}{c}30 \times 50 \\
\text { Bond B eam }\end{array}$} \\
\hline Wall Thickness & \multicolumn{3}{|c|}{$0.5 \mathrm{~m}$} & \multicolumn{3}{|c|}{$0.5 \mathrm{~m}$} & \multicolumn{3}{|c|}{$0.5 \mathrm{~m}$} & \multicolumn{3}{|c|}{$0.5 \mathrm{~m}$} & \multicolumn{2}{|c|}{$0.5 \mathrm{~m}$} & \multicolumn{3}{|c|}{$0.2 \mathrm{~m}$} & \multicolumn{3}{|c|}{$0.2 \mathrm{~m}$} \\
\hline Wall No & $\tau$ & & & $\tau$ & $\tau \mathrm{em}$ & & $\tau$ & $\tau \mathrm{em}$ & & $\tau$ & $\tau \mathrm{em}$ & & $\tau$ & $\tau e m$ & $\tau$ & $\tau \mathrm{em}$ & & $\tau$ & $\tau \mathrm{em}$ & \\
\hline ON9 & 0,16 & & $\mathrm{X}$ & & & $\mathrm{X}$ & & & $x$ & 0,09 & 0,11 & $\sqrt{ }$ & 0,16 & $0,17 \sqrt{ }$ & 0,21 & 0,17 & $7 x$ & 0,16 & 0,10 & \\
\hline P2E1-P2E3 & 0,02 & 13 & $\sqrt{ }$ & 0,02 & 0,11 & $\sqrt{ }$ & 0,00 & 0,11 & V & 0,02 & 0,11 & $\sqrt{ }$ & 0,02 & $0,18 \sqrt{ }$ & 0,02 & 0,19 & $9 \sqrt{ }$ & 0,03 & 0,11 & \\
\hline PSA1-PSA & 02 & & $\sqrt{ }$ & 0,01 & 0,11 & $\sqrt{ }$ & & & $\sqrt{ }$ & 0,01 & 0,11 & $\sqrt{ }$ & 0,02 & $0,18 \sqrt{ }$ & 0,03 & 0,19 & $9 \sqrt{ }$ & 0,01 & 0,11 & $\sqrt{ }$ \\
\hline P2AR1 & 10 & & $\sqrt{ }$ & 0,08 & 0,12 & $\sqrt{ }$ & 0,09 & & 2 & 0,06 & 0,12 & $\sqrt{ }$ & 0,10 & $0,18 \sqrt{ }$ & 0,13 & 0,19 & $9 \sqrt{ }$ & 0,10 & 0,11 & \\
\hline P2YD & 00 & & $\sqrt{ }$ & 0,00 & 0,12 & $\sqrt{ }$ & 0,00 & 0,12 & 2 & 0,00 & 0,12 & $\sqrt{ }$ & 0,00 & $0,19 \sqrt{ }$ & 0,00 & 0,19 & $9 \sqrt{ }$ & 0,01 & 0,12 & \\
\hline & & & $\sqrt{ }$ & & 0,12 & $\sqrt{ }$ & & & $2 \sqrt{ }$ & 0,00 & 0,12 & $\sqrt{ }$ & 0,00 & $19 \sqrt{ }$ & 0,00 & 0,19 & $9 \sqrt{ }$ & 0,00 & 0,12 & \\
\hline & 00 & & $\sqrt{ }$ & 0,00 & 0,12 & $\sqrt{ }$ & 0,00 & 0,12 & $2 \sqrt{ }$ & 0,00 & 0,12 & $\sqrt{ }$ & 0,00 & $0,19 \sqrt{ }$ & 0,00 & 0,19 & $9 \sqrt{ }$ & 0,00 & 0,12 & \\
\hline & & & $\sqrt{ }$ & & 0,12 & $\sqrt{ }$ & & & 2 & 0,08 & 0,11 & $\sqrt{ }$ & 0,06 & $0,18 \sqrt{ }$ & 0,07 & 0,19 & $9 \mathrm{~V}$ & 0,10 & 0,12 & \\
\hline P2IC2 & 09 & 0,13 & $\checkmark$ & 0,08 & 0,12 & $\sqrt{ }$ & 0,09 & 0,12 & $2 \sqrt{ }$ & 0,08 & 0,11 & $\sqrt{ }$ & 0,09 & $0,18 \vee \sqrt{ }$ & 0,11 & 0,19 & $9 \sqrt{ }$ & 0,10 & 0,12 & \\
\hline & & & $\sqrt{ }$ & & & $\sqrt{ }$ & & & V & 0,01 & 0,11 & $\sqrt{ }$ & 0,02 & $0,18 \sqrt{ }$ & 0,03 & 0,19 & $9 \sqrt{ }$ & 0,01 & 0,11 & \\
\hline P2AR1 & 10 & 0,13 & $\checkmark$ & 0,08 & 0,12 & $\sqrt{ }$ & 0,09 & 0,12 & $2 \mathrm{~V}$ & 0,06 & 0,12 & $\sqrt{ }$ & 0,10 & $0,18 \sqrt{ }$ & 0,13 & 0,19 & $9 \sqrt{ }$ & 0,10 & 0,11 & \\
\hline 210 & 00 & 0,14 & $\sqrt{ }$ & & 0,12 & $\sqrt{ }$ & 0,00 & & 2 & 0,00 & 0,12 & $\sqrt{ }$ & 0,00 & $0,19 \sqrt{ }$ & 0,00 & 0,19 & $9 \sqrt{ }$ & 0,01 & 0,12 & \\
\hline $\mathrm{P} 2 \mathrm{YC} 1$ & 00 & 0,14 & $\sqrt{ }$ & 0,00 & 0,12 & $\sqrt{ }$ & 0,00 & 0,12 & 2 & 0,00 & 0,12 & $\sqrt{ }$ & 0,00 & $0,19 \sqrt{ }$ & 0,00 & 0,19 & $9 \mathrm{~V}$ & 0,00 & 0,12 & \\
\hline & & & $\sqrt{ }$ & & 0,12 & $\sqrt{ }$ & & & $2 \sqrt{ }$ & 0,00 & 0,12 & $\sqrt{ }$ & 0,00 & $0,19 \sqrt{ }$ & 0,00 & 0,19 & $9 \mathrm{~V}$ & 0,00 & 0,12 & \\
\hline P2IC1 & 06 & & V & 0,08 & 0,12 & $\sqrt{ }$ & 0,08 & 0,12 & $2 \sqrt{ }$ & 0,08 & 0,11 & $\sqrt{ }$ & 0,06 & $0,18 \sqrt{ }$ & 0,07 & 0,19 & $9 \mathrm{~V}$ & 0,10 & 0,12 & \\
\hline $\mathrm{P} 2 \mathrm{IC} 2$ & 0,09 & 0,13 & $\sqrt{ }$ & 0,08 & 0,12 & $\sqrt{ }$ & 0,09 & 0,12 & $2 \mathrm{~V}$ & 0,08 & 0,11 & $\sqrt{ }$ & 0,09 & $0,18 \sqrt{ }$ & 0,11 & 0,19 & $9 \mathrm{~V}$ & 0,10 & 0,12 & \\
\hline P1ON1-P1ON7 & 0,18 & & $\mathrm{X}$ & 0,12 & 0,13 & $\sqrt{ }$ & 0,15 & 0,13 & $\mathrm{x}$ & 0,09 & 0,13 & $\sqrt{ }$ & 0,18 & $0,19 \sqrt{ }$ & 0,23 & 0,20 & $0 x$ & 0,17 & 0,13 & \\
\hline P1E3 & 0,05 & 0,16 & $\sqrt{ }$ & 0,03 & 0,13 & $\sqrt{ }$ & 0,03 & 0,13 & $\sqrt{ }$ & 0,02 & 0,13 & $\sqrt{ }$ & 0,05 & $0,21 \sqrt{ }$ & 0,02 & 0,22 & $2 \sqrt{ }$ & 0,01 & 0,13 & \\
\hline $\mathrm{P} 1 \mathrm{~A} 1-\mathrm{P} 1 \mathrm{~A} 3$ & 0,04 & & $\sqrt{ }$ & & 0,13 & $\sqrt{ }$ & & 0,13 & $\sqrt{ }$ & 0,01 & 0,13 & $\sqrt{ }$ & 0,04 & $0,21 \mathrm{~V}$ & 0,02 & 0,22 & $2 \sqrt{ }$ & 0,00 & 0,13 & \\
\hline P1AR1-P1AR5 & 0,12 & 0,17 & $\sqrt{ }$ & 0,08 & 0,14 & $\sqrt{ }$ & 0,10 & 0,14 & $+\sqrt{ }$ & 0,06 & 0,14 & $\sqrt{ }$ & 0,12 & $0,21 \sqrt{ }$ & 0,16 & 0,23 & $3 \sqrt{ }$ & 0,11 & 0,14 & \\
\hline P1YD & 0,02 & 0,18 & $\checkmark$ & & 0,15 & $\sqrt{ }$ & & 0,15 & 5 & 0,01 & 0,15 & $\sqrt{ }$ & 0,02 & $0,23 \mathrm{~V}$ & 0,01 & 0,25 & $5 \sqrt{ }$ & 0,01 & 0,15 & \\
\hline $\mathrm{P} 1 \mathrm{YC} 1$ & 0,01 & 0,18 & $\sqrt{ }$ & 0,01 & 0,14 & $\sqrt{ }$ & 0,01 & 0,15 & $5 \sqrt{ }$ & 0,01 & 0,14 & $\sqrt{ }$ & 0,01 & $0,23 \sqrt{ }$ & 0,00 & 0,24 & $4 \sqrt{ }$ & 0,00 & 0,15 & \\
\hline P1YB1 & 0,02 & 0,18 & V & & 0,15 & $\sqrt{ }$ & & 0,15 & $5 \sqrt{ }$ & 0,01 & 0,15 & $\mathrm{~V}$ & 0,02 & $0,23 \sqrt{ }$ & 0,01 & 0,25 & $5 \mathrm{~V}$ & 0,01 & 0,15 & \\
\hline $\mathrm{P} 1 \mathrm{C} 1$ & 0,11 & 0,18 & $\sqrt{ }$ & 0,09 & 0,14 & $\sqrt{ }$ & 0,10 & 0,15 & $5 \mathrm{~V}$ & 0,11 & 0,14 & $\sqrt{ }$ & 0,11 & $0,23 \sqrt{ }$ & 0,14 & 0,24 & $4 \sqrt{ }$ & 0,12 & 0,15 & \\
\hline $\mathrm{P} 1 \mathrm{C} 2$ & 0,12 & 0,18 & $\sqrt{ }$ & 0,09 & 0,14 & $\sqrt{ }$ & 0,10 & 0,15 & $5 \mathrm{~V}$ & 0,11 & 0,14 & $+\sqrt{ }$ & 0,12 & $0,23 \sqrt{ }$ & 0,16 & 0,24 & $4 \sqrt{ }$ & 0,12 & 0,15 & \\
\hline
\end{tabular}
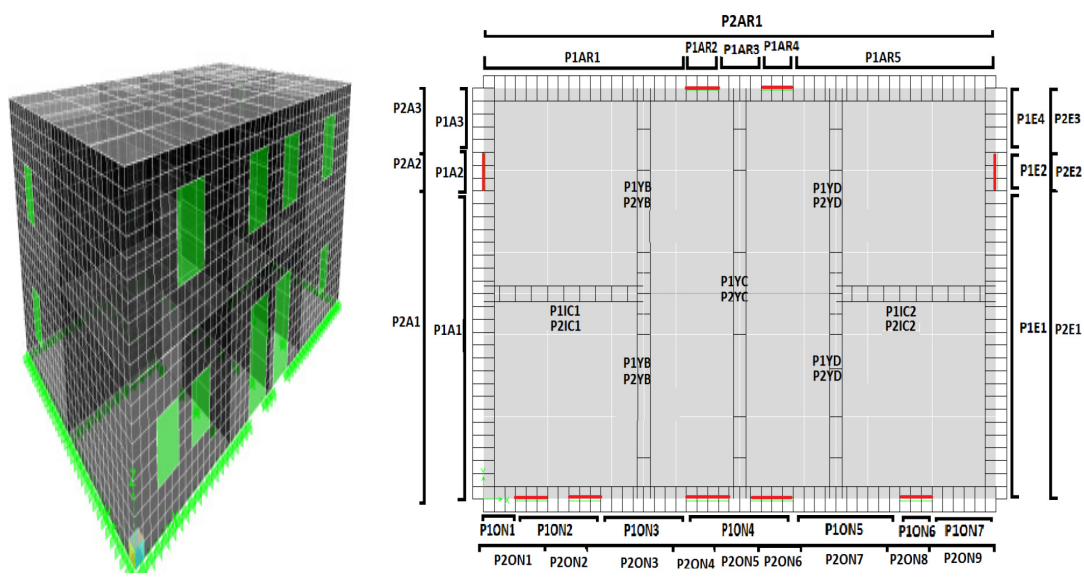

Figure 7: $\quad$ Image and plan of the $2^{\text {nd }}$ model. 
Table 4: $\quad$ Analysis results of the $3^{\text {rd }}$ model.

\begin{tabular}{|c|c|c|c|c|c|c|c|c|c|c|c|c|c|c|}
\hline \multirow{3}{*}{$\begin{array}{c}\text { B uilding Types } \\
\text { Wall Thickness } \\
\text { Wall No } \\
\end{array}$} & \multicolumn{2}{|c|}{ Original } & \multicolumn{2}{|c|}{$\begin{array}{c}50 \times 50 \\
\text { Bond Beam }\end{array}$} & \multicolumn{2}{|c|}{$\begin{array}{c}\text { 30x50 } \\
\text { Bond Beam }\end{array}$} & \multicolumn{2}{|c|}{$\begin{array}{c}\text { 50x50 Bond } \\
\text { Beam }+ \\
\end{array}$} & \multicolumn{2}{|c|}{$\begin{array}{c}\text { Repair } \\
\text { Mortar +FRP }\end{array}$} & \multicolumn{2}{|c|}{$\begin{array}{c}\text { Repair } \\
\text { Mortar +FRP } \\
\end{array}$} & \multicolumn{2}{|c|}{$\begin{array}{c}30 \times 50 \\
\text { Bond Beam }\end{array}$} \\
\hline & \multicolumn{2}{|c|}{$0.5 \mathrm{~m}$} & \multicolumn{2}{|c|}{$0.5 \mathrm{~m}$} & \multicolumn{2}{|c|}{$0.5 \mathrm{~m}$} & \multicolumn{2}{|c|}{$0.5 \mathrm{~m}$} & \multicolumn{2}{|c|}{$0.5 \mathrm{~m}$} & \multicolumn{2}{|c|}{$0.2 \mathrm{~m}$} & \\
\hline & $\tau$ & $\tau \mathrm{em}$ & $\tau$ & $\tau \mathrm{em}$ & $\tau$ & $\tau \mathrm{em}$ & $\tau$ & $\tau \mathrm{em}$ & $\tau$ & $\tau \mathrm{em}$ & $\tau$ & $\tau \mathrm{em}$ & $\tau$ & $\tau \mathrm{em}$ \\
\hline P3A1-P3A3 & 0,03 & $0,13 \sqrt{ }$ & 0,02 & $0,11 \sqrt{ }$ & 0,02 & $0,12 \sqrt{ }$ & 0,02 & $0,11 \sqrt{ }$ & 0,03 & $0,18 \sqrt{ }$ & 0,03 & $0,19 \sqrt{ }$ & 0,02 & $0,11 \sqrt{ }$ \\
\hline P3AR & 0,11 & $0,13 \sqrt{ }$ & 0,08 & $0,12 \sqrt{ }$ & 0,10 & $0,12 \sqrt{ }$ & 0,07 & $0,12 \sqrt{ }$ & 0,11 & $0,18 \sqrt{ }$ & 0,15 & $0,19 \sqrt{ }$ & 10 & $\sqrt{2}$ \\
\hline $\mathrm{P} 3 \mathrm{IC1}$ & 04 & $0,13 \sqrt{ }$ & 0,08 & $0,12 \sqrt{ }$ & 08 & $0,12 \sqrt{ }$ & 05 & $0,11 \sqrt{ }$ & 0,04 & $0,18 \sqrt{ }$ & 0,05 & $\sqrt{1}$ & 0,09 & \\
\hline $\mathrm{P} 3 \mathrm{IC}$ & 09 & $0,13 \sqrt{ }$ & 08 & 0,12 V & 0,09 & 0,12 V & & $0,11 \sqrt{ }$ & 0,09 & & 11 & & 0,09 & \\
\hline $3 \mathrm{ON} 1-$ & 0,18 & $0,12 \mathrm{X}$ & 0,11 & $0,11 \mathrm{X}$ & 0,14 & $0,11 \mathrm{X}$ & 10 & $0,11 \sqrt{ }$ & 0,18 & & 23 & & 0,14 & \\
\hline P3E1 & 02 & $13 \sqrt{ }$ & 0,02 & $11 \mathrm{~V}$ & 0,01 & $0,12 \sqrt{ }$ & 02 & $0,11 \sqrt{ }$ & 0,02 & & 0,02 & & 0,03 & \\
\hline & 00 & & & $1 \sqrt{ }$ & & $12 \mathrm{~V}$ & & 0,11 & 0,00 & & & & 0,00 & \\
\hline & 00 & $\sqrt{ }$ & & $1 \sqrt{ }$ & 00 & $0,12 \sqrt{ }$ & 00 & $0,11 \sqrt{ }$ & 0,00 & & 00 & & 0,00 & \\
\hline P3YB1 & 0,01 & $3 \sqrt{3}$ & 0,01 & $0,11 \sqrt{ }$ & 0,00 & $0,12 \sqrt{ }$ & 0,01 & $0,11 \sqrt{ }$ & 0,01 & $0,18 \sqrt{ }$ & 0,01 & $19 \sqrt{ }$ & 0,00 & $0,11 \sqrt{ }$ \\
\hline 2ON1-P2ON9 & 0,29 & $0,13 X$ & 0,18 & $0,12 X$ & 0,21 & \begin{tabular}{ll|l}
$0,12 X$ \\
\end{tabular} & 0,11 & $0,11 \sqrt{ }$ & 0,29 & $0,18 X$ & 0,36 & $\mathrm{X}$ & 0,20 & $0,12 X$ \\
\hline P2E1-P2E3 & 0,03 & $0,16 \sqrt{ }$ & 0,02 & $0,13 \sqrt{ }$ & 0,01 & $0,13 \sqrt{ }$ & 0,01 & $0,13 \sqrt{ }$ & 0,03 & $0,21 \sqrt{ }$ & 0,03 & $3 \sqrt{ }$ & 0,03 & $0,12 \sqrt{ }$ \\
\hline $\mathrm{P} 2 \mathrm{~A} 1-\mathrm{P} 2 \mathrm{~A} 3$ & 0,04 & $0,16 \sqrt{ }$ & 0,01 & $0,13 \sqrt{ }$ & 0,01 & $0,14 \sqrt{ }$ & 0,02 & $0,13 \sqrt{ }$ & 0,04 & $0,21 \sqrt{ }$ & 0,04 & & 0,01 & $0,13 \sqrt{ }$ \\
\hline & 0,17 & $0,17 \mathrm{X}$ & 0,13 & $0,13 \sqrt{ }$ & 0,15 & $0,14 \mathrm{X}$ & 0,09 & $0,13 \sqrt{ }$ & 0,17 & $0,22 \sqrt{ }$ & 0,21 & $3 \sqrt{1}$ & 0,15 & $0,13 X$ \\
\hline P2YD & 0,00 & $0,17 \sqrt{ }$ & 0,01 & $0,13 \sqrt{ }$ & 0,01 & $0,14 \sqrt{ }$ & 0,01 & $0,13 \sqrt{ }$ & 0,00 & $0,22 \sqrt{ }$ & 0,00 & $0,24 \sqrt{ }$ & 0,01 & $0,13 \sqrt{ }$ \\
\hline & 0,01 & $0,17 \sqrt{ }$ & 0,00 & 0,13 V & 0,00 & $0,14 \sqrt{ }$ & 0,01 & $0,13 \sqrt{ }$ & 0,01 & $0,22 \sqrt{ }$ & 0,00 & $24 \sqrt{ }$ & 0,00 & $13 \sqrt{ }$ \\
\hline & 0,00 & $0,17 \sqrt{ }$ & 01 & $0,13 \sqrt{ }$ & 01 & $0,14 \sqrt{ }$ & 1 & $0,13 \sqrt{ }$ & 0,00 & $0,22 \sqrt{ }$ & 0,01 & $24 \sqrt{ }$ & 0,01 & $13 \mathrm{~V}$ \\
\hline & 0 & $0,17 \sqrt{ }$ & & $0,13 \sqrt{ }$ & 0 , & $0,14 \mathrm{X}$ & & $0,13 \sqrt{ }$ & 0,11 & $0,22 \sqrt{ }$ & 12 & $3 \sqrt{1}$ & 0,14 & \\
\hline & & & & & & $0,14 X$ & & & 0,15 & & & & 0,14 & \\
\hline & 0,18 & $0,21 \sqrt{ }$ & & $0,15 \sqrt{ }$ & 0,13 & $0,16 \sqrt{ }$ & 0,03 & $0,13 \sqrt{ }$ & 0,18 & 0,2 & 0,22 & & 0,11 & \\
\hline $10 \mathrm{N9}$ & 0,26 & $6 \mathrm{X}$ & 0,18 & $0,14 \mathrm{X}$ & 0,22 & $0,14 \mathrm{X}$ & 0,06 & $0,12 \sqrt{ }$ & 0,26 & $0,21 \mathrm{X}$ & 0,33 & $0,23 \mathrm{X}$ & 0,20 & $14 \mathrm{X}$ \\
\hline & 0,08 & $0,19 \sqrt{ }$ & 0,04 & $0,15 \sqrt{ }$ & 0,05 & $0,15 \sqrt{ }$ & 0,03 & $0,15 \sqrt{ }$ & 0,08 & $0,24 \sqrt{ }$ & 03 & $5 \sqrt{1}$ & 0,02 & $15 \sqrt{ }$ \\
\hline P1A1-P1A3 & 0,06 & $0,19 \sqrt{ }$ & 0,03 & $0,15 \sqrt{ }$ & 0,04 & $0,15 \sqrt{ }$ & 0,02 & $0,15 \sqrt{ }$ & 0,06 & $0,24 \sqrt{ }$ & 0,03 & $0,26 \sqrt{ }$ & 0,01 & $0,15 \sqrt{ }$ \\
\hline P1AR1-P1AR5 & 0,18 & $0,17 X$ & 0,12 & $0,13 \sqrt{ }$ & 0,15 & $0,14 X$ & 0,08 & $0,14 \sqrt{ }$ & 0,17 & $0,22 \sqrt{ }$ & 0,21 & $0,23 \sqrt{ }$ & 0,14 & $0,14 \sqrt{ }$ \\
\hline P1YD & 0,03 & $0,22 \sqrt{ }$ & 0,03 & $0,16 \sqrt{ }$ & 0,03 & $0,17 \sqrt{ }$ & 0,01 & $0,16 \sqrt{ }$ & 0,03 & $0,27 \sqrt{ }$ & 0,02 & $0,29 \sqrt{ }$ & 0,01 & $0,17 \sqrt{ }$ \\
\hline P1YC1 & 0,01 & $0,22 \sqrt{ }$ & 0,01 & $0,16 \sqrt{ }$ & 0,01 & $0,17 \sqrt{ }$ & 0,01 & $0,16 \sqrt{ }$ & 0,01 & $0,27 \sqrt{ }$ & 0,00 & $0,29 \sqrt{ }$ & 0,01 & $0,16 \sqrt{ }$ \\
\hline & 0,03 & $0,22 \sqrt{ }$ & 0,03 & $0,16 \sqrt{ }$ & 0,03 & $0,17 \sqrt{ }$ & 0,01 & $0,16 \sqrt{ }$ & 0,03 & $0,27 \sqrt{ }$ & 0,02 & $0,29 \mathrm{~V}$ & 0,01 & $0,17 \mathrm{~V}$ \\
\hline & 0,16 & $0,21 \sqrt{ }$ & 0,13 & $0,16 \sqrt{ }$ & 0,15 & $0,17 \mathrm{~V}$ & 0,12 & $0,16 \sqrt{ }$ & 0,16 & $0,26 \sqrt{ }$ & 0,21 & $0,28 \sqrt{ }$ & 0,15 & $0,16 \sqrt{ }$ \\
\hline $\mathrm{P} 1 \mathrm{IC} 2$ & 0,17 & $0,21 \mathrm{~V}$ & 0,13 & $0,16 \sqrt{ }$ & 0,15 & $0,17 \sqrt{ }$ & 0,12 & $0,16 \sqrt{ }$ & 0,17 & $0,26 \sqrt{ }$ & 0,23 & $0,28 \sqrt{ }$ & 0,15 & $0,16 \sqrt{ }$ \\
\hline
\end{tabular}

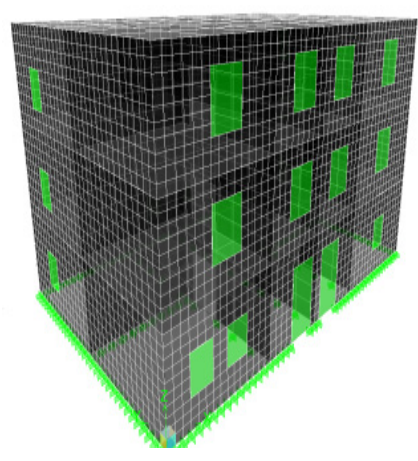

Figure 8: $\quad$ Image and plan of $3^{\text {rd }}$ model.

\section{Conclusion and result}

In order to determine the behaviour of masonry structures, 3 different types of masonry building were analysed in this study. The wall thicknesses of buildings 
were $50 \mathrm{~cm}$. Three of the buildings which were heavily and irregularly constructed were found inadequate in terms of resistance to earthquake and horizontal loads. After adding 30x50 (cm x cm) and 50x50 (cm x cm) vertical bond beams and horizontal bond beams which were bonding other ones to the buildings, then the structures were re-analysed. Although they were less than before, inadequate walls were detected in three of the models. On the other hand, all of the buildings were reinforced with cross steel bars and FRP. In that case, the numbers of inadequate walls were significantly less than that in the original state and that in bond beam-reinforced state. It is thought that when these analyses performed within elasticity limits would be performed according to calculations including plastic deformations, performance levels could be obtained.

As a result; when they are irregular and heavy, those masonry structures cannot satisfy the requirements of code. They need to have horizontal and vertical bond beams and to be regular in architectural aspect. On the other hand, the reinforcement of those kinds of buildings is not accepted as economic. So, for reinforcement of those kinds of houses, historical ones should be considered. Other structures should be demolished and then re-constructed.

\section{References}

[1] Lourenço, P.B., de Borst, R. And Rots, J.G., A Plane Stress Softening Plasticity Model for Orthotropic Materials. International Journal for Numerical Methods in Engineering, Vol.40, No. 21, pp. 4011-4032, 1997.

[2] Lourenço, P.B. and Rots, J.G., Multisurface Interface Model for Analysis of Masonry Structures. Journal of Engineering Mechanics, Vol. 123, No. 7, pp. 660-668, 1997.

[3] Lourenço, P.B., Competitions on Historic Masonry Structures, University of Minho, Portugal, Prog. Structural Engineering Materials, pp. 301-319, 2002.

[4] Varma M.N., Jangid R.S., Achwal V.G., Tension Ring in Masonry Domes, International Conference Structural Analysis of Historical Constructions,1187-1193, New Delhi, India, 2006.

[5] Koçak, A., Demir, A. G., Earthquake Analysis of Historical Church of St Sergius and Bacchus, Istanbul-Turkey. 5th International Conference Structural Analysis of Historical Constructions, Vol.3, 2023-2035, New Delhi, India, 2006.

[6] Eyyubov, C., Uğur, Y., Koçak, A., Eyyubov, İ., The Behavior of Structures Made From Local İngredients During The Destructive Earthquakes. 7th International Congress on Advances in Civil Engineering (ACE2006), Özet: 257-258, Tam Metin: CD-ROM, İstanbul, Türkiye, 2006.

[7] Koçak, A., Köksal, T., Investigation of Earthquake Behavior of the Church of St. Sergius and Bacchus in İstanbul/Turkey. Advanced Materials Research, 133-134, pp. 821-830, 2010.

[8] Koçak, A., Zengin, B., Determining Materials and Free Vibration Values of Historical Structures by Way of Sampling. 8th International Conference on 
Structural Analysis of Historical Constructions (SAHC 2012), pp. 11891204, Wroklaw, Poland, 2012.

[9] Jing M., Raongjant W., Kerdmongkon R., Compressive Strenghening of Damaged Historic Masonry Walls Repaired with GFRP. Advanced Materials Research, 133-134, pp. 965-970, 2010.

[10] Oliveira D.V., Lourenço P.B., Garbin E., Valuzzi M.R., Modena C., Experimental Investigation on the Structural Behavior and Strenthening of Three-leaf Stone Masonry Walls, International Conference Structural Analysis of Historical Constructions, 817-826, New Delhi, India, 2006.

[11] Koçak, A., Köksal, T., An example for determining the cause of damaged in historical buildings: Little Hagio Sophia (Church of St. Sergius and Bacchus)-Istanbul, Turkey. Engineering Failure Analysis, 17 (4), pp. 926937., 2010.

[12] Koçak, A., Zengin, B., Observed Seismic Damages to Historical Structures which are located in the Marmara Region of Turkey and Mechanical Properties of Structures. 8th International Conference on Structural Analysis of Historical Constructions (SAHC 2012), pp. 1599-1611, Wroklaw, Poland, 2012.

[13] Kheyroddin A., Saghafi M.H. and Safakhah S., Strengthening of Historical Masonry Buildings with Fiber Reinforced Polymers(FRP). Advanced Materials Research, 133-134, pp. 903-910, 2010.

[14] Betti M., Orlando M., Vignoli A., Modelling and Analysis of an Italian medieval Castle Under Earthquake loading: Diagnosis and Strengthening, Structural Analysis of Historical Constructions, New Delhi, pp. 1529-1536, 2006.

[15] Angotti F., Aprile L., Orlando M., Ortolani B., Vignoli A., Nonlinear Analysis and Strengthening Design of an Italian Masonry Monumental Building, Structural Analysis of Historical Constructions, New Delhi, pp. 1143-1150.

[16] Önal, M. M., Koçak, A., Methods of Building Damage and Repair and Strengthening of Masonry Details. Congress on Civil Engineering Problems in Antalya region, Vol. I, Antalya, Turkey, pp. 93-108, 2005.

[17] Koçak, A., Tugay, Ş., Kilit, Z., Improving the Earthquake Performance of a Historic Structure and Restoration Work in Antalya. Symposium on Strengthening and Transfer of Historical Monuments in the future with confidence -1, Ankara, Türkiye, pp. 381-395, 2007.

[18] Koçak, A., Kilit, Z., Restoration and Strengthening of a Historical Structure in Antalya - Turkey. Advanced Materials Research, 133-134, pp. 575-584, 2010.

[19] Bayülke, N., The behavior of earthquakes of brick masonry structures. Bulletin of Earthquake Research Institute, 6, pp. 26-41, 1978.

[20] Özmen, T., The Experimental Studies on Stone Masonry Walls. Earthquake Research Bulletin, Year:12, No:49, pp. 68-82, 1985.

[21] Dowrick, D.J., Earthquake Resistant Design, John Wiley and Sons, pp. 142-150, 361-370, 1992. 
[22] Mertol, A. and Mertol, H.C., (eds). Deprem Mühendisliği, Depreme Dayanıklı Yapı Tasarımı, Kozan Ofset, Ankara, 2002.

[23] Budak, A., Uysal, A. and Aydın, C.A., Earthquake Behaviour of Rural Buildings, Atatürk University Journal of the Faculty of Agriculture, Vol. 35, No: 3-4, 2004.

[24] Divleli, A., Elevation Analysis of Traditional Turkish Houses: IstanbulExample of Zeyrek Semti Haydar District, Selçuk University Institute of Science and Master of Science Thesis, Department of Architecture, Konya, 2008.

[25] Aksoy, E., Orta Mekân: Enterprise Architecture Fundamental Principles of the Turkish Civil. Architecture and Art, pp. 7-8, 39-92, 1963.

[26] Tuztaşı U., Formal and Scientific Explanations for the Differentiation of the Idealottoman 'Turkish' House from Anatolian Houses. The Journal of International Social Research, Volume: 3 Issue: 14, 2010.

[27] Baycan, A., Master Degree Thesis, Damage Detection and Evaluation of Masonry Structures, 2004.

[28] Çetin, S., Traditional Residential Architecture Ecological Implications: Burdur Örneği. 5. National Roof \& Side Symposium, 15 -16 April, İzmir, 2010.

[29] Karakaş, S., Karaşin A., Gürbüz Ş., Özyılmaz H., Evaluation of Potential Disaster masonry buildings in Diyarbakır Suriçi, TMMOB Symposium on disaster, 5-7 December, Ankara, pp. 369-374, 2007.

[30] İlgün, Ş., Traditional Yozgat House, Selçuk University Institute of Science and Master of Science Thesis, Department of Architecture, pp. 168, 2007.

[31] Gür, N. V., Deniz, Ö.Ş., Ekinci S., Structural Materials and Components in Masonry Walls. 6. National Roof \& Side Symposium, 2012.

[32] Bayülke, N., Earthquake Response of Masonry Structures and Safety, 1. Conference on Earthquake Engineering and Seismology in Turkey, 2011. 